\title{
Further Results on Resistance Distance and Kirchhoff Index in Electric Networks
}

\author{
Qun Liu, ${ }^{1}$ Jia-Bao Liu, ${ }^{2}$ and Jinde Cao ${ }^{3}$ \\ ${ }^{1}$ Department of Mathematics and Statistics, Lanzhou University, Lanzhou 730000, China \\ ${ }^{2}$ Department of Public Courses, Anhui Xinhua University, Hefei 230088, China \\ ${ }^{3}$ Research Center for Complex Systems and Network Science, Department of Mathematics, Southeast University, \\ Nanjing 210096, China
}

Correspondence should be addressed to Jinde Cao; jdcao@seu.edu.cn

Received 11 September 2015; Revised 31 December 2015; Accepted 6 January 2016

Academic Editor: Juan R. Torregrosa

Copyright (C) 2016 Qun Liu et al. This is an open access article distributed under the Creative Commons Attribution License, which permits unrestricted use, distribution, and reproduction in any medium, provided the original work is properly cited.

\begin{abstract}
In electric circuit theory, it is of great interest to compute the effective resistance between any pairs of vertices of a network, as well as the Kirchhoff index. Let $Q(G)$ be the graph obtained from $G$ by inserting a new vertex into every edge of $G$ and by joining by edges those pairs of these new vertices which lie on adjacent edges of $G$. The set of such new vertices is denoted by $I(G)$. The $Q$-vertex corona of $G_{1}$ and $G_{2}$, denoted by $G_{1} \odot_{Q} G_{2}$, is the graph obtained from vertex disjoint $Q\left(G_{1}\right)$ and $\left|V\left(G_{1}\right)\right|$ copies of $G_{2}$ by joining the $i$ th vertex of $V\left(G_{1}\right)$ to every vertex in the $i$ th copy of $G_{2}$. The Q-edge corona of $G_{1}$ and $G_{2}$, denoted by $G_{1} \ominus_{Q} G_{2}$, is the graph obtained from vertex disjoint $Q\left(G_{1}\right)$ and $\left|I\left(G_{1}\right)\right|$ copies of $G_{2}$ by joining the $i$ th vertex of $I\left(G_{1}\right)$ to every vertex in the $i$ th copy of $G_{2}$. The objective of the present work is to obtain the resistance distance and Kirchhoff index for composite networks such as $Q$-vertex corona and $Q$-edge corona networks.
\end{abstract}

\section{Introduction}

For decades we want to know what a graph looks like. We want to reveal the principles of the networks behaviour covered by their complex topology and dynamics. We want to learn about how the network structure evolves over time and how it affects the properties of the dynamic processes on it. Klein and Randić [1] introduced a new distance function named resistance distance based on electric network theory; the resistance distance between vertices $v_{i}$ and $v_{j}$, denoted by $r_{G}\left(v_{i}, v_{j}\right)$, is defined to be the effective electrical resistance between them if each edge of $G$ is replaced by a unit resistor. Resistance distance is an important character of a graph, which can imply many of its dynamic properties. The Kirchhoff index of $G$ is the sum of resistance distances between all pairs of vertices of $G$. The Kirchhoff index of a network denotes the mean first-passage time measuring the efficiency of random walks; see [1]. The resistance distance and the Kirchhoff index attracted extensive attention due to its wide applications in complex network, physics, chemistry, and others. For more information on resistance distance and
Kirchhoff index of graphs, the readers are referred to [2$22]$ and the references therein. In view of the applications of the resistance distance and the Kirchhoff index, it is of great interest to calculate this parameter for composite networks and find possible relations between the resistance distance and Kirchhoff indexes of the original networks and those of their composite networks; see, for instance, [3].

Let $G=(V(G), E(G))$ be a graph with vertex set $V(G)$ and edge set $E(G)$. Let $d_{i}$ be the degree of vertex $i$ in $G$ and let $D_{G}=\operatorname{diag}\left(d_{1}, d_{2}, \ldots, d_{|V(G)|}\right)$ be the diagonal matrix with all vertex degrees of $G$ as its diagonal entries. For a graph $G$, let $A_{G}$ and $B_{G}$ denote the adjacency matrix and vertex-edge incidence matrix of $G$, respectively. The matrix $L_{G}=D_{G}-A_{G}$ is called the Laplacian matrix of $G$, where $D_{G}$ is the diagonal matrix of vertex degrees of $G$. We use $\mu_{1}(G) \geq u_{2}(G) \geq \cdots \geq$ $\mu_{n}(G)=0$ to denote the eigenvalues of $L_{G}$. Let $l(G)$ be the line graph of $G$.

The $\{1\}$-inverse of $M$ is a matrix $X$ such that $M X M=M$. If $M$ is singular, then it has infinite many $\{1\}$-inverses [6]. We use $M^{(1)}$ to denote any $\{1\}$-inverse of a matrix $M$, and let 

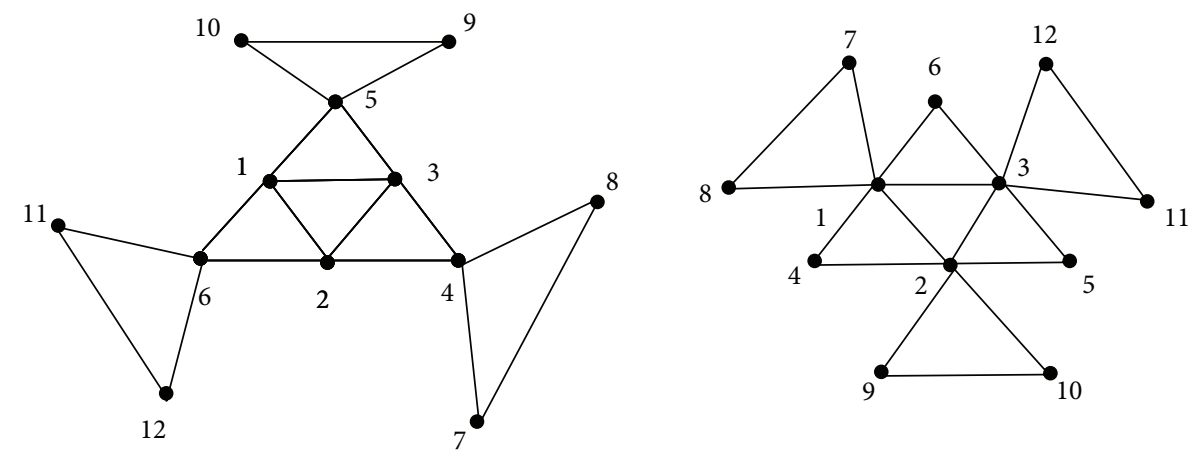

Figure 1: $C_{3} \odot_{\mathrm{Q}} P_{2}$ and $C_{3} \ominus_{\mathrm{Q}} P_{2}$.

$(M)_{u v}$ denote the $(u, v)$-entry of $M$. For a square matrix $M$, the group inverse of $M$, denoted by $M^{\#}$, is the unique matrix $X$ such that $M X M=M, X M X=X$, and $M X=X M$. It is known that $M^{\#}$ exists if and only if $\operatorname{rank}(M)=\operatorname{rank}\left(M^{2}\right)$ $[6,9]$. If $M$ is real symmetric, then $M^{\#}$ exists and $M^{\#}$ is a symmetric $\{1\}$-inverse of $M$. Actually, $M^{\#}$ is equal to the Moore-Penrose inverse of $M$ since $M$ is symmetric [9]. It is known that resistance distances in a connected graph $G$ can be obtained from any $\{1\}$-inverse of $G[7,8]$.

For a graph $G$, let $Q(G)$ be the graph obtained from $G$ by inserting a new vertex into every edge of $G$ and by joining by edges those pairs of these new vertices which lie on adjacent edges of $G$. Let $I(G)$ be the set of newly added vertices; that is, $I(G)=V(Q(G)) \backslash V(G)$. Next, we define two new operations as follows.

Let $G_{1}$ and $G_{2}$ be two vertex-disjoint graphs.

Definition 1. The Q-vertex corona of $G_{1}$ and $G_{2}$, denoted by $G_{1} \odot_{Q} G_{2}$, is the graph obtained from vertex disjoint $Q\left(G_{1}\right)$ and $\left|V\left(G_{1}\right)\right|$ copies of $G_{2}$ by joining the $i$ th vertex of $V\left(G_{1}\right)$ to every vertex in the $i$ th copy of $G_{2}$.

Definition 2. The Q-edge corona of $G_{1}$ and $G_{2}$, denoted by $G_{1} \ominus_{Q} G_{2}$, is the graph obtained from vertex disjoint $Q\left(G_{1}\right)$ and $\left|I\left(G_{1}\right)\right|$ copies of $G_{2}$ by joining the $i$ th vertex of $I\left(G_{1}\right)$ to every vertex in the $i$ th copy of $G_{2}$.

Note that if $G_{i}$ has $n_{i}$ vertices and $m_{i}$ edges for $i=1,2$, then $G_{1} \odot_{Q} G_{2}$ has $n_{1}+m_{1}+n_{1} n_{2}$ vertices and $G_{1} \ominus_{Q} G_{2}$ has $n_{1}+m_{1}+m_{1} n_{2}$ vertices.

Let $P_{n}$ and $C_{n}$ denote a path and cycle with $n$ vertices, respectively. From these definitions, Figure 1 shows the graphs $C_{3} \odot_{\mathrm{Q}} P_{2}$ and $\mathrm{C}_{3} \ominus_{\mathrm{Q}} P_{2}$.

$\mathrm{Bu}$ et al. investigated resistance distance in subdivisionvertex join and subdivision-edge join of graphs [4]. Liu et al. [5] gave the resistance distance and Kirchhoff index of $R$ vertex join and $R$-edge join of two graphs. Motivated by these works, in this paper we will work on two different composite networks: Q-vertex corona and Q-edge corona networks. In fact, we will follow the techniques used in [4] but in a slightly different method in order to obtain the effective resistances and the Kirchhoff indexes of $Q$-vertex corona and Q-edge corona networks in terms of the same parameters on the factors.

\section{Preliminaries}

In this section we list some lemmas as underlying but necessary preliminaries, which will be used in the proofs of our main results.

Lemma 3 (see $[7,9])$. Let $G$ be a connected graph. Then

$$
\begin{aligned}
r_{u v}(G) & =\left(L_{G}^{(1)}\right)_{u u}+\left(L_{G}^{(1)}\right)_{v v}-\left(L_{G}^{(1)}\right)_{u v}-\left(L_{G}^{(1)}\right)_{v u} \\
& =\left(L_{G}^{\#}\right)_{u u}+\left(L_{G}^{\#}\right)_{v v}-2\left(L_{G}^{\#}\right)_{u v} .
\end{aligned}
$$

Let $1_{n}$ denote the column vector of dimension $n$ with all the entries equal to one. We will often use 1 to denote an allones column vector if the dimension can be read from the context.

Lemma 4 (see [4]). For any graph, one has $L_{G}^{\#} 1=0$.

Lemma 5 (see [17]). Let

$$
M=\left(\begin{array}{ll}
A & B \\
C & D
\end{array}\right)
$$

be a nonsingular matrix. If $A$ and $D$ are nonsingular, then

$$
\begin{aligned}
M^{-1} & =\left(\begin{array}{cc}
A^{-1}+A^{-1} B S^{-1} C A^{-1} & -A^{-1} B S^{-1} \\
-S^{-1} C A^{-1} & S^{-1}
\end{array}\right) \\
& =\left(\begin{array}{cc}
\left(A-B D^{-1} C\right)^{-1} & -A^{-1} B S^{-1} \\
-S^{-1} C A^{-1} & S^{-1}
\end{array}\right),
\end{aligned}
$$

where $S=D-C A^{-1} B$.

For a square matrix $M$, let $\operatorname{tr}(M)$ denote the trace of $M$.

Lemma 6 (see [10]). Let $G$ be a connected graph on $n$ vertices. Then

$$
\mathrm{Kf}(G)=n \operatorname{tr}\left(L_{G}^{(1)}\right)-1^{T} L_{G}^{(1)} 1=n \operatorname{tr}\left(L_{G}^{\#}\right) .
$$

Lemma 7. Let

$$
L=\left(\begin{array}{cc}
A & B \\
B^{T} & D
\end{array}\right)
$$


be the Laplacian matrix of a connected graph. If $D$ is nonsingular, then

$$
X=\left(\begin{array}{cc}
H^{\#} & -H^{\#} B D^{-1} \\
-D^{-1} B^{T} H^{\#} & D^{-1}+D^{-1} B^{T} H^{\#} B D^{-1}
\end{array}\right)
$$

is a symmetric $\{1\}$-inverse of $L$, where $H=A-B D^{-1} B^{T}$.

Proof. Since $H=A-B D^{-1} B^{T}$ is symmetric, $H^{\#}$ exists and is symmetric. Since

$$
L=\left(\begin{array}{cc}
I & B D^{-1} \\
0 & I
\end{array}\right)\left(\begin{array}{cc}
H & 0 \\
0 & D
\end{array}\right)\left(\begin{array}{cc}
I & 0 \\
D^{-1} B^{T} & I
\end{array}\right)
$$

we know that

$$
X=\left(\begin{array}{cc}
I & 0 \\
-D^{-1} B^{T} & I
\end{array}\right)\left(\begin{array}{cc}
H^{\#} & 0 \\
0 & D^{-1}
\end{array}\right)\left(\begin{array}{cc}
I & -B D^{-1} \\
0 & I
\end{array}\right)
$$

is a symmetric $\{1\}$-inverse of $L$.

Remarks 1. The above result is similar to Lemma 2.8 in [10]; this is another form of Lemma 2.8, but in the process of computing the resistance distance of graphs $G_{1} \odot_{Q} G_{2}$ and $G_{1} \ominus_{Q} G_{2}$, we use this formula to be more superior than Lemma 2.8 in [10].

\section{Resistance Distance in Q-Vertex Coronae and $Q$-Edge Coronae of Two Graphs}

We first give explicit resistance distance for the arbitrary twovertex resistance distance in $G_{1} \odot_{\mathrm{Q}} G_{2}$.

Theorem 8. Let $G_{1}$ be an $r_{1}$-regular graph on $n_{1}$ vertices and $m_{1}$ edges, and let $G_{2}$ be an arbitrary graph on $n_{2}$ vertices and $m_{2}$ edges. Then the following holds:

(i) For any e, $f \in E\left(G_{1}\right)$, one has

$$
r_{e f}\left(G_{1} \odot_{\mathrm{Q}} G_{2}\right)=\frac{r_{1}}{1+r_{1}} r_{e f}\left(l\left(G_{1}\right)\right) .
$$

(ii) For any $u, v \in V\left(G_{2}\right)$, one has

$$
\begin{aligned}
r_{u v}\left(G_{1} \odot_{\mathrm{Q}} G_{2}\right) & \\
= & \left(I_{n_{1}} \otimes\left(L_{G_{2}}+I_{n_{2}}-\frac{1}{n_{2}+r_{1}} j_{n_{2} \times n_{2}}\right)^{-1}\right)_{u u} \\
& +\left(I_{n_{1}} \otimes\left(L_{G_{2}}+I_{n_{2}}-\frac{1}{n_{2}+r_{1}} j_{n_{2} \times n_{2}}\right)^{-1}\right)_{v v} \\
& -2\left(I_{n_{1}} \otimes\left(L_{G_{2}}+I_{n_{2}}-\frac{1}{n_{2}+r_{1}} j_{n_{2} \times n_{2}}\right)^{-1}\right)_{u v} .
\end{aligned}
$$

(iii) For any $u \in V\left(G_{1}\right)$, let $e_{1}, e_{2}, \ldots, e_{r}$ be $r$ edges incident to $u$ in $G_{1}$. For any $u \in V\left(G_{1}\right), f \in E\left(G_{1}\right)$, one has

$$
\begin{aligned}
r_{u f}\left(G_{1} \odot_{Q} G_{2}\right)= & \left(\frac{1}{r_{1}} I_{n_{1} \times n_{1}}+\frac{1}{r_{1}\left(1+r_{1}\right)} R L_{l\left(G_{1}\right)}^{\#} R^{T}\right)_{u u} \\
& +\frac{r_{1}}{1+r_{1}}\left(L_{l\left(G_{1}\right)}^{\#}\right)_{f f} \\
& -\frac{2}{r_{1}\left(1+r_{1}\right)}\left(R L_{l\left(G_{1}\right)}^{\#}\right)_{u f} .
\end{aligned}
$$

(iv) For any $u, v \in V\left(G_{1}\right)(u \neq v)$, let $e_{1}, e_{2}, \ldots, e_{r}$ (resp., $f_{1}, f_{2}, \ldots, f_{r}$ ) be $r$ edges incident to $u$ (resp., $v$ ) in $G_{1}$, one has

$$
\begin{aligned}
r_{u v}\left(G_{1} \odot_{\mathrm{Q}} G_{2}\right)= & \frac{2}{r_{1}}+\frac{1}{r_{1}\left(r_{1}+1\right)}\left(R L_{l\left(G_{1}\right)}^{\#} R^{T}\right)_{u u} \\
& +\frac{1}{r_{1}\left(r_{1}+1\right)}\left(R L_{l\left(G_{1}\right)}^{\#} R^{T}\right)_{v v} \\
& -\frac{2}{r_{1}+1}\left(R L_{l\left(G_{1}\right)}^{\#} R^{T}\right)_{u v} .
\end{aligned}
$$

(v) For any $u \in V\left(G_{1}\right)$, let $e_{1}, e_{2}, \ldots, e_{r}$ be $r$ edges incident to $u$ in $G_{1}$. For any $u \in V\left(G_{1}\right), v \in V\left(G_{2}\right)$, one has

$$
\begin{aligned}
r_{u v}\left(G_{1} \odot_{Q} G_{2}\right) & =\frac{1}{r_{1}}+\frac{1}{r_{1}\left(r_{1}+1\right)}\left(R L_{l\left(G_{1}\right)}^{\#} R^{T}\right)_{u u} \\
& +\left(I_{n_{1}} \otimes\left(L_{G_{2}}+I_{n_{2}}-\frac{1}{n_{2}+r_{1}} j_{n_{2} \times n_{2}}\right)^{-1}\right)_{v v} \\
& -2\left(I_{n_{1}} \otimes\left(L_{G_{2}}+I_{n_{2}}-\frac{1}{n_{2}+r_{1}} j_{n_{2} \times n_{2}}\right)^{-1}\right)_{u v} .
\end{aligned}
$$

(vi) For any e $\in E\left(G_{1}\right), v \in V\left(G_{2}\right)$,

$$
\begin{aligned}
r_{e v}\left(G_{1} \odot_{\mathrm{Q}} G_{2}\right) & \\
= & \frac{r_{1}}{1+r_{1}}\left(L_{l\left(G_{1}\right)}^{\#}\right)_{e e} \\
& +\left(I_{n_{1}} \otimes\left(L_{G_{2}}+I_{n_{2}}-\frac{1}{n_{2}+r_{1}} j_{n_{2} \times n_{2}}\right)^{-1}\right)_{v v} \\
& -\frac{2}{1+r_{1}}\left(R L_{l\left(G_{1}\right)}^{\#}\right)_{e v} .
\end{aligned}
$$

Proof. Let $B$ and $R$ be the adjacency matrix of $l\left(G_{1}\right)$ and incidence matrix of $G_{1}$, respectively. Then the Laplacian matrix of $G_{1} \odot_{\mathrm{Q}} G_{2}$ is as follows:

$$
\begin{aligned}
& L\left(G_{1} \odot_{Q} G_{2}\right) \\
& =\left(\begin{array}{ccc}
2 r_{1} I_{m_{1}}-B & -R^{T} & 0_{m_{1} \times n_{1} n_{2}} \\
-R & \left(r_{1}+n_{2}\right) I_{n_{1} \times n_{1}} & -I_{n_{1}} \otimes 1_{n_{2}}^{T} \\
0_{n_{1} n_{2} \times m_{1}} & -I_{n_{1}} \otimes 1_{n_{2}} & I_{n_{1}} \otimes\left(L_{\left(G_{2}\right)}+I_{n_{2}}\right)
\end{array}\right) .
\end{aligned}
$$


Let $A=2 r_{1} I_{m_{1}}-B, B=\left(\begin{array}{ll}-R^{T} & 0_{m_{1} \times n_{1} n_{2}}\end{array}\right), B^{T}=\left(\begin{array}{c}-R \\ 0_{n_{1} n_{2} \times m_{1}}\end{array}\right)$, and $\quad D^{-1}$

$$
D=\left(\begin{array}{cc}
\left(r_{1}+n_{2}\right) I_{n_{1} \times n_{1}} & -I_{n_{1}} \otimes 1_{n_{2}}^{T} \\
-I_{n_{1}} \otimes 1_{n_{2}} & I_{n_{1}} \otimes\left(L_{\left(G_{2}\right)}+I_{n_{2}}\right)
\end{array}\right) . \quad(16) \quad=\left(\begin{array}{cc}
\frac{1}{r_{1}} I_{n_{1} \times n_{1}} & \frac{1}{r_{1}}\left(I_{n_{1}} \otimes 1_{n_{2}}^{T}\right) \\
\frac{1}{r_{1}}\left(I_{n_{1}} \otimes 1_{n_{2}}\right) & I_{n_{1}} \otimes\left(L_{G_{2}}+I_{n_{2}}-\frac{1}{n_{2}+r_{1}} j_{n_{2} \times n_{2}}\right)^{-1}
\end{array}\right) .
$$

Note that $R R^{T}=D_{G_{1}}+A_{G_{1}}$. Let $R_{1}=I_{n_{1}} \otimes\left(L\left(G_{2}\right)+I_{n_{2}}\right)-$ $\left(-I_{n_{1}} \otimes 1_{n_{2}}\right)\left(\left(r_{1}+n_{2}\right) I_{n_{1} \times n_{1}}\right)^{-1}\left(-I_{n_{1}} \otimes 1_{n_{2}}^{T}\right)=I_{n_{1}} \otimes\left(L_{G_{2}}+I_{n_{2}}-\right.$ $\left.\left(1 /\left(n_{2}+r_{1}\right)\right) j_{n_{2} \times n_{2}}\right)^{-1}$. By Lemma 5 , we have

Now we are ready to calculate $\{1\}$-inverse of $L\left(G_{1} \odot_{\mathrm{Q}} G_{2}\right)$. First we begin with the calculation about $H$.

Let $Q=I_{n_{1}} \otimes\left(L_{G_{2}}+I_{n_{2}}-\left(1 /\left(n_{2}+r_{1}\right)\right) j_{n_{2} \times n_{2}}\right)$; then

$$
\begin{aligned}
H & =2 r_{1} I_{m_{1}}-B-\left(\begin{array}{ll}
-R^{T} & 0_{m_{1} \times n_{1} n_{2}}
\end{array}\right)\left(\begin{array}{cc}
\frac{1}{r_{1}} I_{n_{1}} & \frac{1}{r_{1}}\left(I_{n_{1}} \otimes 1_{n_{2}}^{T}\right) \\
\frac{1}{r_{1}}\left(I_{n_{1}} \otimes 1_{n_{2}}\right) & Q^{-1}
\end{array}\right)\left(\begin{array}{c}
-R \\
0_{n_{1} n_{2} \times m_{1}}
\end{array}\right) \\
& =2 r_{1} I_{m_{1}}-B-\left(-\frac{1}{r} R^{T}-\frac{1}{r_{1}} R^{T}\left(I_{n_{1}} \otimes 1_{n_{2}}^{T}\right)\right)\left(\begin{array}{c}
-R \\
0_{n_{1} n_{2} \times m_{1}}
\end{array}\right)=2 r_{1} I_{m_{1}}-B-\frac{1}{r_{1}}\left(2 I_{m_{1}}+B\right)=\frac{1+r_{1}}{r_{1}} L_{l\left(G_{1}\right)} .
\end{aligned}
$$

By Lemma 7 , we have $H^{\#}=\left(r_{1} /\left(1+r_{1}\right)\right) L_{l\left(G_{1}\right)}^{\#}$.

Next according to Lemma 7, we calculate $-H^{\#} B D^{-1}$ and $-D^{-1} B^{T} H^{\#}$ :

$$
-H^{\#} B D^{-1}=-\frac{r_{1}}{1+r_{1}}
$$$$
\cdot L_{l\left(G_{1}\right)}^{\#}\left(\begin{array}{ll}
-R^{T} & 0_{m_{1} \times n_{1} n_{2}}
\end{array}\right)\left(\begin{array}{cc}
\frac{1}{r_{1}} I_{n_{1}} & \frac{1}{r_{1}}\left(I_{n_{1}} \otimes 1_{n_{2}}^{T}\right) \\
\frac{1}{r_{1}}\left(I_{n_{1}} \otimes 1_{n_{2}}\right) & Q^{-1}
\end{array}\right)
$$$$
=-\frac{r_{1}}{1+r_{1}} L_{l\left(G_{1}\right)}^{\#}\left(-\frac{1}{r_{1}} R^{T}-\frac{1}{r_{1}} R^{T}\left(I_{n_{1}} \otimes 1_{n_{2}}^{T}\right)\right)=\frac{1}{1+r_{1}}
$$$$
\cdot\left(L_{l\left(G_{1}\right)}^{\#} R^{T} L_{l\left(G_{1}\right)}^{\#} R^{T}\left(I_{n_{1}} \otimes 1_{n_{2}}^{T}\right)\right),
$$$$
-D^{-1} B^{T} H^{\#}
$$

$$
=-\left(\begin{array}{cc}
\frac{1}{r_{1}} I_{n_{1}} & \frac{1}{r_{1}}\left(I_{n_{1}} \otimes 1_{n_{2}}^{T}\right) \\
\frac{1}{r_{1}}\left(I_{n_{1}} \otimes 1_{n_{2}}\right) & Q^{-1}
\end{array}\right)\left(\begin{array}{c}
-R \\
0_{n_{1} n_{2} \times m_{1}}
\end{array}\right) \frac{r_{1}}{1+r_{1}}
$$

$$
\begin{aligned}
& \cdot L_{l\left(G_{1}\right)}^{\#}=\left(\begin{array}{c}
\frac{1}{r_{1}} R \\
\frac{1}{r_{1}}\left(I_{n_{1}} \otimes 1_{n_{2}}\right) R
\end{array}\right) \frac{r_{1}}{1+r_{1}} L_{l\left(G_{1}\right)}^{\#}=\frac{1}{1+r_{1}} \\
& \cdot\left(\begin{array}{c}
R L_{l\left(G_{1}\right)}^{\#} \\
\left(I_{n_{1}} \otimes 1_{n_{2}}\right) R L_{l\left(G_{1}\right)}^{\#}
\end{array}\right) .
\end{aligned}
$$

We are ready to compute $D^{-1} B^{T} H^{\#} B D^{-1}$. then

$$
\text { Let } K=I_{n_{1}} \otimes 1_{n_{2}}, Q=I_{n_{1}} \otimes\left(L_{G_{2}}+I_{n_{2}}-\left(1 /\left(n_{2}+r_{1}\right)\right) j_{n_{2} \times n_{2}}\right)^{-1} \text {; }
$$

$$
\begin{aligned}
& D^{-1} B^{T} H^{\#} B D^{-1}=-\frac{1}{1+r_{1}} \\
& \cdot\left(\begin{array}{c}
R L_{l\left(G_{1}\right)}^{\#} \\
K R L_{l\left(G_{1}\right)}^{\#}
\end{array}\right)\left(\begin{array}{ll}
-R^{T} & 0_{m_{1} \times n_{1} n_{2}}
\end{array}\right)\left(\begin{array}{cc}
\frac{1}{r_{1}} I_{n_{1} \times n_{1}} & \frac{1}{r_{1}} K^{T} \\
\frac{1}{r_{1}} K & Q^{-1}
\end{array}\right) \\
& =-\frac{1}{1+r_{1}}\left(\begin{array}{c}
R L_{l\left(G_{1}\right)}^{\#} \\
K R L_{l\left(G_{1}\right)}^{\#}
\end{array}\right)\left(-\frac{1}{r_{1}} R^{T}-\frac{1}{r_{1}} R^{T} K^{T}\right) \\
& =\left(\begin{array}{cc}
\frac{1}{r_{1}\left(1+r_{1}\right)} R L_{l\left(G_{1}\right)}^{\#} R^{T} & \frac{1}{r_{1}\left(1+r_{1}\right)} R L_{l\left(G_{1}\right)}^{\#} R^{T} K^{T} \\
\frac{1}{r_{1}\left(1+r_{1}\right)} K R L_{l\left(G_{1}\right)}^{\#} R^{T} & \frac{1}{r_{1}\left(1+r_{1}\right)} K R L_{l\left(G_{1}\right)}^{\#} R^{T} K^{T}
\end{array}\right) .
\end{aligned}
$$

Based on Lemmas 5 and 7, the following matrix

$$
N=\left(\begin{array}{ccc}
\frac{r_{1}}{1+r_{1}} L_{l\left(G_{1}\right)}^{\#} & \frac{1}{1+r_{1}} L_{l\left(G_{1}\right)}^{\#} R^{T} & \frac{1}{1+r_{1}} L_{l\left(G_{1}\right)}^{\#} R^{T} K^{T} \\
\frac{1}{1+r_{1}} R L_{l\left(G_{1}\right)}^{\#} & \frac{1}{r_{1}} I_{n_{1} \times n_{1}}+\frac{1}{r_{1}\left(1+r_{1}\right)} R L_{l\left(G_{1}\right)}^{\#} R^{T} & \frac{1}{r_{1}\left(1+r_{1}\right)} R L_{l\left(G_{1}\right)}^{\#} R^{T} K^{T}+\frac{1}{r_{1}} K^{T} \\
\frac{1}{1+r_{1}} K R L_{l\left(G_{1}\right)}^{\#} & \frac{1}{r_{1}} K+\frac{1}{r_{1}\left(1+r_{1}\right)} K R L_{l\left(G_{1}\right)}^{\#} R^{T} & Q^{-1}+\frac{1}{r_{1}\left(1+r_{1}\right)} K R L_{l\left(G_{1}\right)}^{\#} R^{T} K^{T}
\end{array}\right)
$$


is a symmetric $\{1\}$-inverse of $L\left(G_{1} \odot_{Q} G_{2}\right)$, where $K=I_{n_{1}} \otimes 1_{n_{2}}$ and $Q=I_{n_{1}} \otimes\left(L_{G_{2}}+I_{n_{2}}-\left(1 /\left(n_{2}+r_{1}\right)\right) j_{n_{2} \times n_{2}}\right)$.

For any e, $f \in E\left(G_{1}\right)$, by Lemma 3 and (21), we have

$$
r_{e f}\left(G_{1} \odot_{Q} G_{2}\right)=\frac{r_{1}}{1+r_{1}} r_{e f}\left(l\left(G_{1}\right)\right) .
$$

For any $u, v \in V\left(G_{2}\right)$, by Lemma 3 and (21), we have

$$
\begin{aligned}
r_{u v}( & \left.G_{1} \odot_{Q} G_{2}\right) \\
= & \left(I_{n_{1}} \otimes\left(L_{G_{2}}+I_{n_{2}}-\frac{1}{n_{2}+r_{1}} j_{n_{2} \times n_{2}}\right)^{-1}\right)_{u u} \\
& +\left(I_{n_{1}} \otimes\left(L_{G_{2}}+I_{n_{2}}-\frac{1}{n_{2}+r_{1}} j_{n_{2} \times n_{2}}\right)^{-1}\right)_{v v} \\
& -2\left(I_{n_{1}} \otimes\left(L_{G_{2}}+I_{n_{2}}-\frac{1}{n_{2}+r_{1}} j_{n_{2} \times n_{2}}\right)^{-1}\right)_{u v} .
\end{aligned}
$$

For any $u \in V\left(G_{1}\right)$, let $e_{1}, e_{2}, \ldots, e_{r}$ be $r$ edges incident to $u$ in $G_{1}$. For any $u \in V\left(G_{1}\right), f \in E\left(G_{1}\right)$, by Lemma 3 and (21), we have

$$
\begin{aligned}
r_{u f}\left(G_{1} \odot_{Q} G_{2}\right) & \\
= & \left(\frac{1}{r_{1}} I_{n_{1} \times n_{1}}+\frac{1}{r_{1}\left(1+r_{1}\right)} R L_{l\left(G_{1}\right)}^{\#} R^{T}\right)_{u u} \\
& +\frac{r_{1}}{1+r_{1}}\left(L_{l\left(G_{1}\right)}^{\#}\right)_{f f}-\frac{2}{1+r_{1}}\left(R L_{l\left(G_{1}\right)}^{\#}\right)_{u f} .
\end{aligned}
$$

For any $u, v \in V\left(G_{1}\right)(u \neq v)$, let $e_{1}, e_{2}, \ldots, e_{r}$ (resp., $f_{1}, f_{2}, \ldots, f_{r}$ ) be $r$ edges incident to $u$ (resp., $v$ ) in $G_{1}$. By Lemma 3 and (21), we have

$$
\begin{aligned}
r_{u v}\left(G_{1} \odot_{Q} G_{2}\right)= & \frac{2}{r_{1}}+\frac{1}{r_{1}\left(r_{1}+1\right)}\left(R L_{l\left(G_{1}\right)}^{\#} R^{T}\right)_{u u} \\
& +\frac{1}{r_{1}\left(r_{1}+1\right)}\left(R L_{l\left(G_{1}\right)}^{\#} R^{T}\right)_{v v} \\
& -\frac{2}{r_{1}\left(r_{1}+1\right)}\left(R L_{l\left(G_{1}\right)}^{\#} R^{T}\right)_{u v} .
\end{aligned}
$$

For any $u \in V\left(G_{1}\right)$, let $e_{1}, e_{2}, \ldots, e_{r}$ be $r$ edges incident to $u$ in $G_{1}$. For any $u \in V\left(G_{1}\right), v \in V\left(G_{2}\right)$, by Lemma 3 and (21), we have

$$
\begin{aligned}
r_{u v}\left(G_{1} \odot_{Q} G_{2}\right) & \\
= & \frac{1}{r_{1}}+\frac{1}{r_{1}\left(r_{1}+1\right)}\left(R L_{l\left(G_{1}\right)}^{\#} R^{T}\right)_{u u} \\
& +\left(I_{n_{1}} \otimes\left(L_{G_{2}}+I_{n_{2}}-\frac{1}{n_{2}+r_{1}} j_{n_{2} \times n_{2}}\right)^{-1}\right)_{v v} \\
& -2\left(I_{n_{1}} \otimes\left(L_{G_{2}}+I_{n_{2}}-\frac{1}{n_{2}+r_{1}} j_{n_{2} \times n_{2}}\right)^{-1}\right)_{u v} .
\end{aligned}
$$

For any $e \in E\left(G_{1}\right), v \in V\left(G_{2}\right)$, by Lemma 3 and (21), we have

$$
\begin{aligned}
r_{e v}\left(G_{1} \odot_{\mathrm{Q}} G_{2}\right) & \\
= & \frac{r_{1}}{1+r_{1}}\left(L_{l\left(G_{1}\right)}^{\#}\right)_{e e} \\
& +\left(I_{n_{1}} \otimes\left(L_{G_{2}}+I_{n_{2}}-\frac{1}{n_{2}+r_{1}} j_{n_{2} \times n_{2}}\right)^{-1}\right)_{v v} \\
& -\frac{2}{1+r_{1}}\left(R L_{l\left(G_{1}\right)}^{\#}\right)_{e v} .
\end{aligned}
$$

Next it will give the formulae for resistance distance of graph $G_{1} \ominus_{\mathrm{Q}} G_{2}$ when $G_{1}$ is a regular graph as follows.

Theorem 9. Let $G_{1}$ be an $r_{1}$-regular graph with $n_{1}$ vertices, and let $G_{2}$ be an arbitrary graph with $n_{2}$ vertices. Then the following holds:

(i) For any e, $f \in E\left(G_{1}\right)$, one has

$$
r_{e f}\left(G_{1} \ominus_{Q} G_{2}\right)=\frac{r_{1}}{1+r_{1}} r_{e f}\left(l\left(G_{1}\right)\right)
$$

(ii) For any $u, v \in V\left(G_{2}\right)$, one has

$$
\begin{aligned}
r_{u v}\left(G_{1} \ominus_{Q} G_{2}\right)= & \left(I_{m_{1}} \otimes\left(L_{\left(G_{2}\right)}+I_{n_{2}}\right)^{-1}\right)_{u u} \\
& +\left(I_{m_{1}} \otimes\left(L_{\left(G_{2}\right)}+I_{n_{2}}\right)^{-1}\right)_{v v} \\
& -2\left(I_{m_{1}} \otimes\left(L_{\left(G_{2}\right)}+I_{n_{2}}\right)^{-1}\right)_{u v} .
\end{aligned}
$$

(iii) For any $u \in V\left(G_{1}\right)$, let $e_{1}, e_{2}, \ldots, e_{r}$ be $r$ edges incident to $u$ in $G$. For any $u \in V\left(G_{1}\right), f \in E\left(G_{1}\right)$, one has

$$
\begin{aligned}
r_{u f}\left(G_{1} \ominus_{\mathrm{Q}} G_{2}\right) & \\
= & \left(\frac{1}{r_{1}} I_{n_{1} \times n_{1}}+\frac{1}{r_{1}\left(1+r_{1}\right)} R L_{l\left(G_{1}\right)}^{\#} R^{T}\right)_{u u} \\
& +\frac{r_{1}}{1+r_{1}}\left(L_{l\left(G_{1}\right)}^{\#}\right)_{f f}-\frac{2}{1+r_{1}}\left(R L_{l\left(G_{1}\right)}^{\#}\right)_{u f} .
\end{aligned}
$$

(iv) For any $u, v \in V\left(G_{1}\right)(u \neq v)$, let $e_{1}, e_{2}, \ldots, e_{r}$ (resp., $f_{1}, f_{2}, \ldots, f_{r}$ ) be $r$ edges incident to $u$ (resp., $v$ ) in $G_{1}$; one has

$$
\begin{aligned}
r_{u v}\left(G_{1} \ominus_{\mathrm{Q}} G_{2}\right)= & \frac{2}{r_{1}}+\frac{1}{r_{1}\left(r_{1}+1\right)}\left(R L_{l\left(G_{1}\right)}^{\#} R^{T}\right)_{u u} \\
& +\frac{1}{r_{1}\left(r_{1}+1\right)}\left(R L_{l\left(G_{1}\right)}^{\#} R^{T}\right)_{v v} \\
& -\frac{2}{r_{1}\left(r_{1}+1\right)}\left(R L_{l\left(G_{1}\right)}^{\#} R^{T}\right)_{u v} .
\end{aligned}
$$


(v) For any $u \in V\left(G_{1}\right)$, let $e_{1}, e_{2}, \ldots, e_{r}$ be $r$ edges incident to $u$ in $G_{1}$. For any $u \in V\left(G_{1}\right), v \in V\left(G_{2}\right)$, one has

$$
\begin{aligned}
r_{u v}\left(G_{1} \ominus_{\mathrm{Q}} G_{2}\right)= & \frac{1}{r_{1}}+\frac{1}{r_{1}\left(r_{1}+1\right)}\left(R L_{l\left(G_{1}\right)}^{\#} R^{T}\right)_{u u} \\
& +\left(I_{m_{1}} \otimes\left(L_{\left(G_{2}\right)}+I_{n_{2}}\right)^{-1}\right)_{v v} \\
& -2\left(I_{m_{1}} \otimes\left(L_{\left(G_{2}\right)}+I_{n_{2}}\right)^{-1}\right)_{u v} .
\end{aligned}
$$

(vi) For any e $\in E\left(G_{1}\right), v \in V\left(G_{2}\right)$, one has

$$
\begin{aligned}
r_{e v}\left(G_{1} \ominus_{Q} G_{2}\right)= & \frac{r_{1}}{1+r_{1}}\left(L_{l\left(G_{1}\right)}^{\#}\right)_{e e} \\
& +\left(I_{m_{1}} \otimes\left(L_{\left(G_{2}\right)}+I_{n_{2}}\right)^{-1}\right)_{v v} \\
& -\frac{2}{1+r_{1}}\left(R L_{l\left(G_{1}\right)}^{\#}\right)_{e v} .
\end{aligned}
$$

Proof. Let $B$ and $R$ be the adjacency matrix of $l\left(G_{1}\right)$ and incidence matrix of $G_{1}$, respectively. Then the Laplacian matrix of $G_{1} \ominus_{\mathrm{Q}} G_{2}$ is as follows:

$$
\begin{aligned}
& L\left(G_{1} \ominus_{\mathrm{Q}} G_{2}\right) \\
& =\left(\begin{array}{ccc}
\left(2 r_{1}+n_{2}\right) I_{m_{1}}-B & -R^{T} & -I_{m_{1}} \otimes 1_{n_{2}}^{T} \\
-R & r_{1} I_{n_{1}} & 0_{n_{1} \times n_{2} m_{1}} \\
-I_{m_{1}} \otimes 1_{n_{2}} & 0_{n_{2} m_{1} \times n_{1}} & I_{m_{1}} \otimes\left(L_{\left(G_{2}\right)}+I_{n_{2}}\right)
\end{array}\right) .
\end{aligned}
$$

Now we are ready to calculate a $\{1\}$-inverse of $L\left(G_{1} \ominus_{Q} G_{2}\right)$.

First we are ready to calculate $H^{\#}$.

Let $M=I_{m_{1}} \otimes 1_{n_{2}}, P=I_{m_{1}} \otimes\left(L_{\left(G_{2}\right)}+I_{n_{2}}\right)$, then

$$
\begin{aligned}
& H=\left(2 r_{1}+n_{2}\right) I_{m_{1}}-B \\
& -\left(\begin{array}{ll}
-R^{T} & -M^{T}
\end{array}\right)\left(\begin{array}{cc}
r_{1} I_{n_{1}} & 0_{n_{1} \times n_{2} m_{1}} \\
0_{n_{2} m_{1} \times n_{1}} & P
\end{array}\right)^{-1}\left(\begin{array}{c}
-R \\
-M
\end{array}\right) \\
& =\left(2 r_{1}+n_{2}\right) I_{m_{1}}-B \\
& -\left(\begin{array}{cc}
-\frac{1}{r_{1}} R^{T} & -M^{T} P^{-1}
\end{array}\right)\left(\begin{array}{c}
-R \\
-M
\end{array}\right) \\
& =\left(2 r_{1}+n_{2}\right) I_{m_{1}}-B-\frac{1}{r_{1}} R^{T} R-n_{2} I_{m_{1}} \\
& =\frac{1+r_{1}}{r_{1}} L_{l\left(G_{1}\right)} .
\end{aligned}
$$

By Lemma 7, we have $H^{\#}=\left(r_{1} /\left(1+r_{1}\right)\right) L_{l\left(G_{1}\right)}^{\#}$.

Next we compute $-H^{\#} B D^{-1}$ and $-D^{-1} B^{T} H^{\#}$ :

$$
\begin{aligned}
& -H^{\#} B D^{-1}=-\frac{r_{1}}{1+r_{1}} \\
& \cdot L_{l\left(G_{1}\right)}^{\#}\left(-R^{T}-I_{m_{1}} \otimes 1_{n_{2}}^{T}\right)\left(\begin{array}{cc}
\frac{1}{r_{1}} I_{n_{1}} & 0_{n_{1} \times n_{2} m_{1}} \\
0_{n_{2} m_{1} \times n_{1}} & I_{m_{1}} \otimes\left(L_{\left(G_{2}\right)}+I_{n_{2}}\right)^{-1}
\end{array}\right) \\
& =-\frac{r_{1}}{1+r_{1}} L_{l\left(G_{1}\right)}^{\#}\left(-\frac{1}{r_{1}} R^{T}-I_{m_{1}} \otimes 1_{n_{2}}^{T}\right) \\
& =\left(\frac{1}{r_{1}+1} L_{l\left(G_{1}\right)}^{\#} R^{T} \frac{r_{1}}{r_{1}+1} L_{l\left(G_{1}\right)}^{\#}\left(I_{m_{1}} \otimes 1_{n_{2}}^{T}\right)\right), \\
& -D^{-1} B^{T} H^{\#} \\
& =-\left(\begin{array}{cc}
\frac{1}{r_{1}} I_{n_{1}} & 0_{n_{1} \times n_{2} m_{1}} \\
0_{n_{2} m_{1} \times n_{1}} & I_{m_{1}} \otimes\left(L_{\left(G_{2}\right)}+I_{n_{2}}\right)^{-1}
\end{array}\right)\left(\begin{array}{c}
-R \\
-I_{m_{1}} \otimes 1_{n_{2}}
\end{array}\right) \frac{r_{1}}{1+r_{1}} \\
& \cdot L_{l\left(G_{1}\right)}^{\#}=\frac{r_{1}}{1+r_{1}}\left(\begin{array}{c}
\frac{1}{r_{1}} R \\
I_{m_{1}} \otimes 1_{n_{2}}
\end{array}\right) L_{l\left(G_{1}\right)}^{\#} \\
& =\left(\begin{array}{c}
\frac{1}{r_{1}+1} R L_{l\left(G_{1}\right)}^{\#} \\
\frac{r_{1}}{r_{1}+1}\left(I_{m_{1}} \otimes 1_{n_{2}}\right) L_{l\left(G_{1}\right)}^{\#}
\end{array}\right) \text {. }
\end{aligned}
$$

We are ready to compute $D^{-1} B^{T} H^{\#} B D^{-1}$ :

$$
\begin{aligned}
& D^{-1} B^{T} H^{\#} B D^{-1} \\
& =-\left(\begin{array}{l}
\frac{1}{r_{1}+1} R L_{l\left(G_{1}\right)}^{\#} \\
\frac{r_{1}}{r_{1}+1} M L_{l\left(G_{1}\right)}^{\#}
\end{array}\right)\left(\begin{array}{ll}
-R^{T} & -M^{T}
\end{array}\right)\left(\begin{array}{cc}
\frac{1}{r_{1}} I_{n_{1}} & 0_{n_{1} \times n_{2} m_{1}} \\
0_{n_{2} m_{1} \times n_{1}} & P^{-1}
\end{array}\right) \\
& =-\left(\begin{array}{l}
\frac{1}{r_{1}+1} R L_{l\left(G_{1}\right)}^{\#} \\
\frac{r_{1}}{r_{1}+1} M L_{l\left(G_{1}\right)}^{\#}
\end{array}\right)\left(\begin{array}{ll}
-\frac{1}{r_{1}} R^{T} & -M^{T} P^{-1}
\end{array}\right) \\
& =\left(\begin{array}{cc}
\frac{1}{r_{1}\left(r_{1}+1\right)} R L_{l\left(G_{1}\right)}^{\#} R^{T} & \frac{1}{r_{1}+1} R L_{l\left(G_{1}\right)}^{\#} M^{T} \\
\frac{1}{r_{1}+1} M L_{l\left(G_{1}\right)}^{\#} R^{T} & \frac{r_{1}}{r_{1}+1} M L_{l\left(G_{1}\right)}^{\#} M^{T}
\end{array}\right) .
\end{aligned}
$$

Based on Lemmas 5 and 7, the following matrix

$$
N=\left(\begin{array}{ccc}
\frac{r_{1}}{1+r_{1}} L_{l\left(G_{1}\right)}^{\#} & \frac{1}{r_{1}+1} L_{l\left(G_{1}\right)}^{\#} R^{T} & \frac{r_{1}}{r_{1}+1} L_{l\left(G_{1}\right)}^{\#} M^{T} \\
\frac{1}{1+r_{1}} R L_{l\left(G_{1}\right)}^{\#} & \frac{1}{r_{1}} I_{n_{1}}+\frac{1}{r_{1}\left(r_{1}+1\right)} R L_{l\left(G_{1}\right)}^{\#} R^{T} & \frac{1}{r_{1}+1} R L_{l\left(G_{1}\right)}^{\#} M^{T} \\
\frac{1}{1+r_{1}} M L_{l\left(G_{1}\right)}^{\#} & \frac{1}{r_{1}+1} M L_{l\left(G_{1}\right)}^{\#} R^{T} & P^{-1}+\frac{r_{1}}{r_{1}+1} M L_{l\left(G_{1}\right)}^{\#} M^{T}
\end{array}\right)
$$


is a symmetric $\{1\}$-inverse of $L\left(G_{1} \ominus_{Q} G_{2}\right)$, where $M=I_{m_{1}} \otimes 1_{n_{2}}$ and $P=I_{m_{1}} \otimes\left(L_{\left(G_{2}\right)}+I_{n_{2}}\right)$.

For any $e, f \in E\left(G_{1}\right)$, by Lemma 3 and (38), we have

$$
r_{e f}\left(G_{1} \ominus_{Q} G_{2}\right)=\frac{r_{1}}{1+r_{1}} r_{e f}\left(l\left(G_{1}\right)\right) .
$$

For any $u, v \in V\left(G_{2}\right)$, by Lemma 3 and (38), we have

$$
\begin{aligned}
r_{u v}\left(G_{1} \ominus_{Q} G_{2}\right)= & \left(I_{m_{1}} \otimes\left(L_{\left(G_{2}\right)}+I_{n_{2}}\right)^{-1}\right)_{u u} \\
& +\left(I_{m_{1}} \otimes\left(L_{\left(G_{2}\right)}+I_{n_{2}}\right)^{-1}\right)_{v v} \\
& -2\left(I_{m_{1}} \otimes\left(L_{\left(G_{2}\right)}+I_{n_{2}}\right)^{-1}\right)_{u v} .
\end{aligned}
$$

For any $u \in V\left(G_{1}\right)$, let $e_{1}, e_{2}, \ldots, e_{r}$ be $r$ edges incident to $u$ in $G$. For any $u \in V\left(G_{1}\right), f \in E\left(G_{1}\right)$, by Lemma 3 and (38), we have

$$
\begin{aligned}
r_{u f}\left(G_{1} \ominus_{Q} G_{2}\right)= & \left(\frac{1}{r_{1}} I_{n_{1} \times n_{1}}+\frac{1}{r_{1}\left(1+r_{1}\right)} R L_{l\left(G_{1}\right)}^{\#} R^{T}\right)_{u u} \\
& +\frac{r_{1}}{1+r_{1}}\left(L_{l\left(G_{1}\right)}^{\#}\right)_{f f} \\
& -\frac{2}{1+r_{1}}\left(R L_{l\left(G_{1}\right)}^{\#}\right)_{u f} .
\end{aligned}
$$

For any $u, v \in V\left(G_{1}\right)(u \neq v)$, let $e_{1}, e_{2}, \ldots, e_{r}$ (resp., $f_{1}, f_{2}, \ldots, f_{r}$ ) be $r$ edges incident to $u$ (resp., $v$ ) in $G_{1}$. By Lemma 3 and (38), we have

$$
\begin{aligned}
r_{u v}\left(G_{1} \ominus_{\mathrm{Q}} G_{2}\right)= & \frac{2}{r_{1}}+\frac{1}{r_{1}\left(r_{1}+1\right)}\left(R L_{l\left(G_{1}\right)}^{\#} R^{T}\right)_{u u} \\
& +\frac{1}{r_{1}\left(r_{1}+1\right)}\left(R L_{l\left(G_{1}\right)}^{\#} R^{T}\right)_{v v} \\
& -\frac{2}{r_{1}\left(r_{1}+1\right)}\left(R L_{l\left(G_{1}\right)}^{\#} R^{T}\right)_{u v} .
\end{aligned}
$$

For any $u \in V\left(G_{1}\right)$, let $e_{1}, e_{2}, \ldots, e_{r}$ be $r$ edges incident to $u$ in $G_{1}$. For any $u \in V\left(G_{1}\right), v \in V\left(G_{2}\right)$, by Lemma 3 and (38), we have

$$
\begin{aligned}
r_{u v}\left(G_{1} \ominus_{Q} G_{2}\right)= & \frac{1}{r_{1}}+\frac{1}{r_{1}\left(r_{1}+1\right)}\left(R L_{l\left(G_{1}\right)}^{\#} R^{T}\right)_{u u} \\
& +\left(I_{m_{1}} \otimes\left(L_{\left(G_{2}\right)}+I_{n_{2}}\right)^{-1}\right)_{v v} \\
& -2\left(I_{m_{1}} \otimes\left(L_{\left(G_{2}\right)}+I_{n_{2}}\right)^{-1}\right)_{u v} .
\end{aligned}
$$
have

For any $e \in E\left(G_{1}\right), v \in V\left(G_{2}\right)$, by Lemma 3 and (38), we

$$
\begin{aligned}
r_{e v}\left(G_{1} \ominus_{Q} G_{2}\right)= & \frac{r_{1}}{1+r_{1}}\left(L_{l\left(G_{1}\right)}^{\#}\right)_{e e} \\
& +\left(I_{m_{1}} \otimes\left(L_{\left(G_{2}\right)}+I_{n_{2}}\right)^{-1}\right)_{v v} \\
& -\frac{2}{1+r_{1}}\left(R L_{l\left(G_{1}\right)}^{\#}\right)_{e v} .
\end{aligned}
$$

\section{Kirchhoff Index in Q-Vertex Corona and Q-Edge Corona of Two Graphs}

Theorem 10. Let $G_{1}$ be an $r_{1}$-regular graph on $n_{1}$ vertices and $m_{1}$ edges, and let $G_{2}$ be an arbitrary graph on $n_{2}$ vertices and $m_{2}$ edges. Then the following holds:

$$
\begin{aligned}
\mathrm{Kf} & \left(G_{1} \odot_{\mathrm{Q}} G_{2}\right)=\left(n_{1}+m_{1}+n_{1} n_{2}\right)\left(\frac{r_{1}}{1+r_{1}} \operatorname{tr}\left(L_{l\left(G_{1}\right)}^{\#}\right)\right. \\
& +\frac{n_{1}}{r_{1}} I_{n_{1}}+\frac{1}{r_{1}\left(1+r_{1}\right)} \operatorname{tr}\left(R L_{l\left(G_{1}\right)}^{\#} R^{T}\right) \\
& +n_{1} \sum_{i=1}^{n_{2}} \frac{1}{\mu_{i}\left(G_{2}\right)+1}+\frac{1}{r_{1}\left(r_{1}+1\right)} \operatorname{tr}\left[\left(I_{n_{1}} \otimes 1_{n_{2}}\right)\right. \\
& \left.\left.\cdot R L_{l\left(G_{1}\right)}^{\#} R^{T}\left(I_{n_{1}} \otimes 1_{n_{2}}^{T}\right)\right]\right)-\frac{n_{1}}{r_{1}}-3 n_{1} n_{2} .
\end{aligned}
$$

Proof. Let $L_{G_{1} \odot_{\mathrm{Q}} G_{2}}^{(1)}$ be the symmetric $\{1\}$-inverse of $L_{G_{1} \odot_{\mathrm{Q}} G_{2}}$. Then

$$
\begin{aligned}
& \operatorname{tr}\left(L_{G_{1} \odot_{Q} G_{2}}^{(1)}\right)=\frac{r_{1}}{1+r_{1}} \operatorname{tr}\left(L_{l\left(G_{1}\right)}^{\#}\right) \\
& +\operatorname{tr}\left(\frac{1}{r_{1}} I_{n_{1}}+\frac{1}{r_{1}\left(1+r_{1}\right)} R L_{l\left(G_{1}\right)}^{\#} R^{T}\right) \\
& +\operatorname{tr}\left(I_{n_{1}} \otimes\left(L_{G_{2}}+I_{n_{2}}-\frac{1}{n_{2}+r_{1}} j_{n_{2} \times n_{2}}\right)^{-1}\right) \\
& +\frac{1}{r_{1}\left(r_{1}+1\right)} \operatorname{tr}\left[\left(I_{n_{1}} \otimes 1_{n_{2}}\right) R L_{l\left(G_{1}\right)}^{\#} R^{T}\left(I_{n_{1}} \otimes 1_{n_{2}}^{T}\right)\right] .
\end{aligned}
$$

Note that the eigenvalues of $\left(L_{G_{2}}+I_{n_{2}}-\left(1 /\left(n_{2}+r_{1}\right)\right) j_{n_{2} \times n_{2}}\right)$ are $\mu_{1}\left(G_{2}\right)+1, \mu_{2}\left(G_{2}\right)+1, \ldots, \mu_{n_{2}}\left(G_{2}\right)+1$. Then

$$
\begin{aligned}
& \operatorname{tr}\left(I_{n_{1}} \otimes\left(L_{G_{2}}+I_{n_{2}}-\frac{1}{n_{2}+r_{1}} j_{n_{2} \times n_{2}}\right)\right) \\
& \quad=n_{1} \sum_{i=1}^{n_{2}}\left(\mu_{i}\left(G_{2}\right)+1\right)^{-1}=n_{1} \sum_{i=1}^{n_{2}} \frac{1}{\mu_{i}\left(G_{2}\right)+1} .
\end{aligned}
$$

So

$$
\begin{aligned}
& \operatorname{tr}\left(L_{G_{1} \odot_{Q} G_{2}}^{(1)}\right)=\frac{r_{1}}{1+r_{1}} \operatorname{tr}\left(L_{l\left(G_{1}\right)}^{\#}\right)+\frac{n_{1}}{r_{1}} I_{n_{1}} \\
& +\frac{1}{r_{1}\left(1+r_{1}\right)} \operatorname{tr}\left(R L_{l\left(G_{1}\right)}^{\#} R^{T}\right)+n_{1} \sum_{i=1}^{n_{2}} \frac{1}{\mu_{i}\left(G_{2}\right)+1} \\
& +\frac{1}{r_{1}\left(r_{1}+1\right)} \operatorname{tr}\left[\left(I_{n_{1}} \otimes 1_{n_{2}}\right) R L_{l\left(G_{1}\right)}^{\#} R^{T}\left(I_{n_{1}} \otimes 1_{n_{2}}^{T}\right)\right] .
\end{aligned}
$$


Next, we calculate $1^{T}\left(L_{G_{1} \odot_{Q} G_{2}}^{(1)}\right) 1$. Since, by Lemma 4 , $L_{G}^{\#} 1=0$, then

$$
\begin{aligned}
& 1^{T}\left(L_{G_{1} \odot_{Q} G_{2}}^{(1)}\right) 1=1^{T}\left(\frac{1}{r_{1}} I_{n_{1}}+\frac{1}{r_{1}\left(1+r_{1}\right)} R L_{l\left(G_{1}\right)}^{\#} R^{T}\right) \\
& \cdot 1+\frac{1}{r_{1}\left(1+r_{1}\right)} 1^{T}\left[R L_{l\left(G_{1}\right)}^{\#} R^{T}\left(I_{n_{1}} \otimes 1_{n_{2}}^{T}\right)\right. \\
& \left.+\frac{1}{r_{1}}\left(I_{n_{1}} \otimes 1_{n_{2}}^{T}\right)\right] 1+1^{T}\left[\frac{1}{r_{1}}\left(I_{n_{1}} \otimes 1_{n_{2}}\right)\right. \\
& \left.+\frac{1}{r_{1}\left(1+r_{1}\right)}\left(I_{n_{1}} \otimes 1_{n_{2}}\right) R L_{l\left(G_{1}\right)}^{\#} R^{T}\right] 1+1^{T}\left(Q^{-1}\right. \\
& \left.+\frac{1}{r_{1}\left(1+r_{1}\right)}\left(I_{n_{1}} \otimes 1_{n_{2}}\right) R L_{l\left(G_{1}\right)}^{\#} R^{T}\left(I_{n_{1}} \otimes 1_{n_{2}}^{T}\right)\right) 1,
\end{aligned}
$$

where $Q=L_{G_{2}}+I_{n_{2}}-\left(1 /\left(n_{2}+r_{1}\right)\right) j_{n_{2} \times n_{2}}$. Since $1^{T} R=R^{T} 1=$ $2 \cdot 1$, then

$$
\begin{aligned}
& \begin{aligned}
1^{T} R L_{l\left(G_{1}\right)}^{\#} R^{T} 1 & =1^{T}\left(R L_{l\left(G_{1}\right)}^{\#} R^{T}\left(I_{m_{1}} \otimes 1_{n_{2}}^{T}\right)\right) 1 \\
& =1^{T}\left(I_{n_{1}} \otimes 1_{n_{2}}^{T}\right) R L_{l\left(G_{1}\right)}^{\#} R^{T} 1 \\
& =1^{T}\left(I_{n_{1}} \otimes 1_{n_{2}}\right) R L_{l\left(G_{1}\right)}^{\#} R^{T}\left(I_{n_{1}} \otimes 1_{n_{2}}^{T}\right) 1 \\
& =0
\end{aligned} \\
& \text { Let } T=1_{n_{1} n_{2}}^{T}\left(I_{n_{1}} \otimes\left(L_{G_{2}}+I_{n_{2}}-\left(1 /\left(n_{2}+r_{1}\right)\right) j_{n_{2} \times n_{2}}\right)^{-1}\right) 1_{n_{2} n_{1}}
\end{aligned}
$$

$$
\begin{aligned}
& T=\left(\begin{array}{llll}
1_{n_{2}}^{T} & 1_{n_{2}}^{T} & \cdots & 1_{n_{2}}^{T}
\end{array}\right)\left(\begin{array}{cccc}
Q^{-1} & & & \\
& Q^{-1} & & \\
& & \ddots & \\
& & & Q^{-1}
\end{array}\right)\left(\begin{array}{c}
1_{n_{2}} \\
1_{n_{2}} \\
\vdots \\
1_{n_{2}}
\end{array}\right) \\
& =\left(1_{n_{2}}^{T}\left(L_{G_{2}}+I_{n_{2}}-\frac{1}{n_{2}+r_{1}} j_{n_{2} \times n_{2}}\right)^{-1} \cdots 1_{n_{2}}^{T}\left(L_{G_{2}}+I_{n_{2}}-\frac{1}{n_{2}+r_{1}} j_{n_{2} \times n_{2}}\right)^{-1}\right)\left(\begin{array}{c}
1_{n_{2}} \\
1_{n_{2}} \\
\vdots \\
1_{n_{2}}
\end{array}\right) \\
& =n_{1} 1_{n_{2}}^{T}\left(L_{G_{2}}+I_{n_{2}}-\frac{1}{n_{2}+r_{1}} j_{n_{2} \times n_{2}}\right)^{-1} 1_{n_{2}}=n_{1} n_{2} .
\end{aligned}
$$

So

$$
1^{T}\left(L_{G_{1} \odot_{Q} G_{2}}^{(1)}\right) 1=\frac{n_{1}}{r_{1}}+3 n_{1} n_{2} .
$$

Lemma 6 implies that

$$
\begin{aligned}
\operatorname{Kf}\left(L_{G_{1} \odot_{Q} G_{2}}^{(1)}\right)= & \left(n_{1}+m_{1}+n_{1} n_{2}\right) \operatorname{tr}\left(L_{G_{1}}^{\#}\right) \\
& -1^{T}\left(L_{G_{1} \odot_{Q} G_{2}}^{(1)}\right) 1 .
\end{aligned}
$$

Then plugging $\operatorname{tr}\left(L_{G_{1} \odot_{\mathrm{Q}} G_{2}}^{(1)}\right)$ and $1^{T}\left(L_{G_{1} \odot_{\mathrm{Q}} G_{2}}^{(1)}\right) 1$ into the equation above, we obtain the required result.
Theorem 11. Let $G_{1}$ be an $r_{1}$-regular graph with $n_{1}$ vertices, and let $G_{2}$ be an arbitrary graph with $n_{2}$ vertices. Then

$$
\begin{aligned}
\operatorname{Kf} & \left(G_{1} \ominus_{Q} G_{2}\right)=\left(n_{1}+m_{1}+m_{1} n_{2}\right)\left(\frac{r_{1}}{1+r_{1}} \operatorname{tr}\left(L_{l\left(G_{1}\right)}^{\#}\right)\right. \\
& +\frac{n_{2}}{r_{1}}+\frac{1}{r_{1}\left(r_{1}+1\right)} \operatorname{tr}\left(R L_{G_{1}}^{\#} R^{T}\right) \\
& +m_{1} \sum_{i=1}^{n_{2}} \frac{1}{\mu_{i}\left(G_{2}\right)+2} \\
& \left.+\frac{1}{1+r_{1}} \operatorname{tr}\left[\left(I_{m_{1}} \otimes 1_{n_{2}}\right) L_{l\left(G_{1}\right)}^{\#}\left(I_{m_{1}} \otimes 1_{n_{2}}^{T}\right)\right]\right)-\frac{n_{1}}{r_{1}} \\
& -n_{1} n_{2} .
\end{aligned}
$$

Proof. The proof is similar to those of Theorem 10, omitted. 


\section{Conflict of Interests}

The authors declare that there is no conflict of interests regarding the publication of this paper.

\section{Acknowledgments}

This work was supported by the National Natural Science Foundation of China (nos. 11461020, 11561042), the Youth Foundation of Hexi University in Gansu Province (no. QN2013-07), and the Teaching Reform Project of Hexi University (no. HXXXJY-2014-011). The work of Jia-Bao Liu was partly supported by the Natural Science Foundation for the Higher Education Institutions of Anhui Province of China under Grant no. KJ2015A331.

\section{References}

[1] D. J. Klein and M. Randić, "Resistance distance," Journal of Mathematical Chemistry, vol. 12, no. 1, pp. 81-95, 1993.

[2] D. J. Klein, "Resistance-distance sum rules," Croatica Chemica Acta, vol. 75, no. 2, pp. 633-649, 2002.

[3] E. Bendito, A. Carmona, and A. M. Encinas, "The Kirchhoff indices of join networks," Discrete Applied Mathematics, vol. 160, no. 1-2, pp. 24-37, 2012.

[4] C. Bu, B. Yan, X. Zhou, and J. Zhou, "Resistance distance in subdivision-vertex join and subdivision-edge join of graphs," Linear Algebra and Its Applications, vol. 458, pp. 454-462, 2014.

[5] X. Liu, J. Zhou, and C. Bu, "Resistance distance and Kirchhoff index of $R$-vertex join and R-edge join of two graphs," Discrete Applied Mathematics, vol. 187, pp. 130-139, 2015.

[6] A. Ben-Israel and T. N. E. Greville, Generalized Inverses: Theory and Applications, Springer, New York, NY, USA, 2nd edition, 2003.

[7] R. B. Bapat, Graphs and Matrices, Universitext, Springer, London, UK; Hindustan Book Agency, New Delhi, India, 2010.

[8] R. B. Bapat and S. Gupta, "Resistance distance in wheels and fans," Indian Journal of Pure and Applied Mathematics, vol. 41, no. 1, pp. 1-13, 2010.

[9] C. Bu, L. Sun, J. Zhou, and Y. Wei, "A note on block representations of the group inverse of Laplacian matrices," Electronic Journal of Linear Algebra, vol. 23, pp. 866-876, 2012.

[10] L. Sun, W. Wang, J. Zhou, and C. Bu, "Some results on resistance distances and resistance matrices," Linear and Multilinear Algebra, vol. 63, no. 3, pp. 523-533, 2015.

[11] H. Chen and F. Zhang, "Resistance distance and the normalized Laplacian spectrum," Discrete Applied Mathematics, vol. 155, no. 5, pp. 654-661, 2007.

[12] W. Xiao and I. Gutman, "Relations between resistance and Laplacian matrices and their applications," MATCH Communications in Mathematical and in Computer Chemistry, vol. 51, pp. 119-127, 2004.

[13] W. Xiao and I. Gutman, "Resistance distance and Laplacian spectrum," Theoretical Chemistry Accounts, vol. 110, no. 4, pp. 284-289, 2003.

[14] Y. Yang and D. J. Klein, "A recursion formula for resistance distances and its applications," Discrete Applied Mathematics, vol. 161, no. 16-17, pp. 2702-2715, 2013.

[15] R. B. Bapat, "Resistance matrix of a weighted graph," MATCH Communications in Mathematical and in Computer, vol. 50, pp. 73-82, 2004.
[16] Y. Yang and D. J. Klein, "Resistance distance-based graph invariants of subdivisions and triangulations of graphs," Discrete Applied Mathematics, vol. 181, pp. 260-274, 2015.

[17] F. Zhang, The Schur Complement and Its Applications, Springer, New York, NY, USA, 2005.

[18] J.-B. Liu, X.-F. Pan, L. Yu, and D. Li, "Complete characterization of bicyclic graphs with minimal Kirchhoff index," Discrete Applied Mathematics, vol. 200, pp. 95-107, 2016.

[19] J.-B. Liu, X.-F. Pan, F.-T. Hu, and F.-F. Hu, "Asymptotic Laplacian-energy-like invariant of lattices," Applied Mathematics and Computation, vol. 253, pp. 205-214, 2015.

[20] J. B. Liu and J. Cao, "The resistance distances of electrical networks based on Laplacian generalized inverse," Neurocomputing, vol. 167, pp. 306-313, 2015.

[21] J.-B. Liu and X.-F. Pan, "A unified approach to the asymptotic topological indices of various lattices," Applied Mathematics and Computation, vol. 270, pp. 62-73, 2015.

[22] Q. Liu, J. B. Liu, and J. Cao, "The Laplacian polynomial and Kirchhoff index of graphs based on R-graphs," Neurocomputing, 2015. 


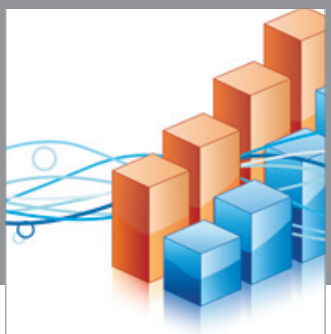

Advances in

Operations Research

vatem alat4

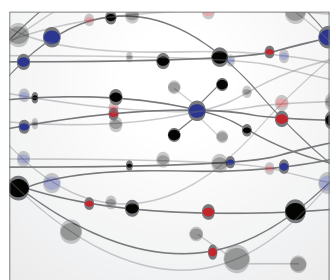

\section{The Scientific} World Journal
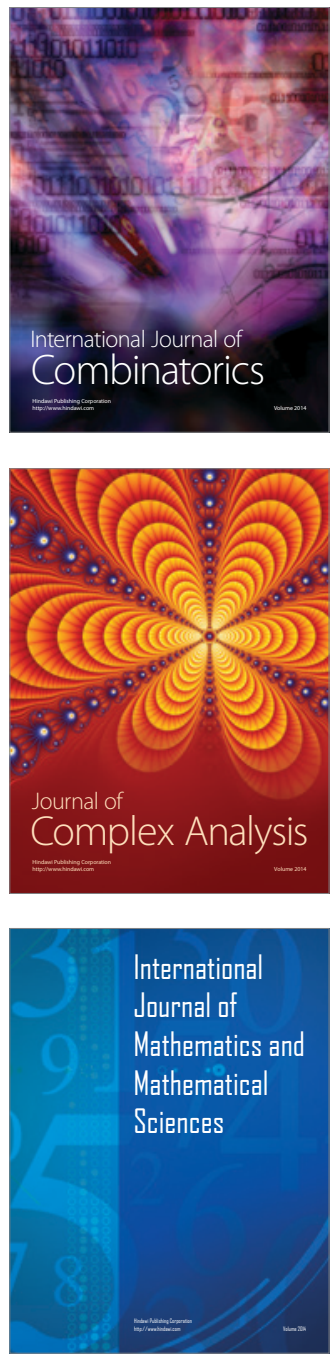
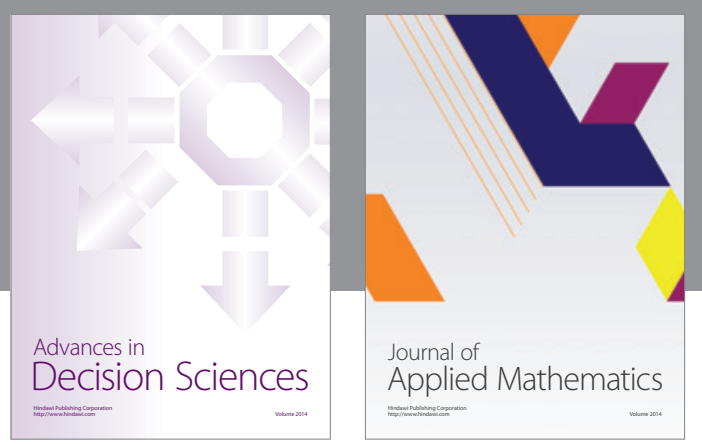

Algebra

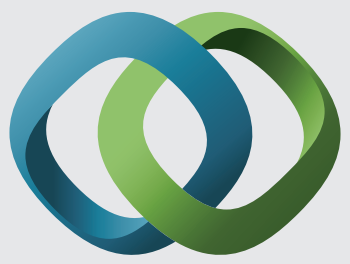

\section{Hindawi}

Submit your manuscripts at

http://www.hindawi.com
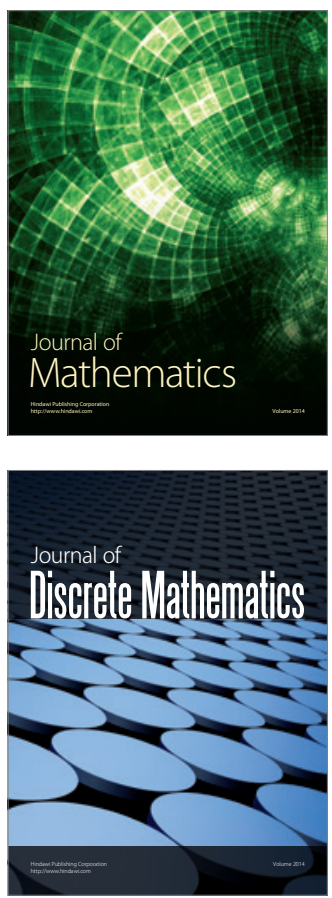

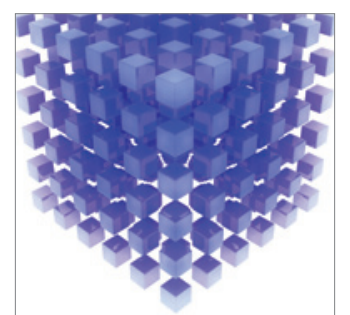

Mathematical Problems in Engineering
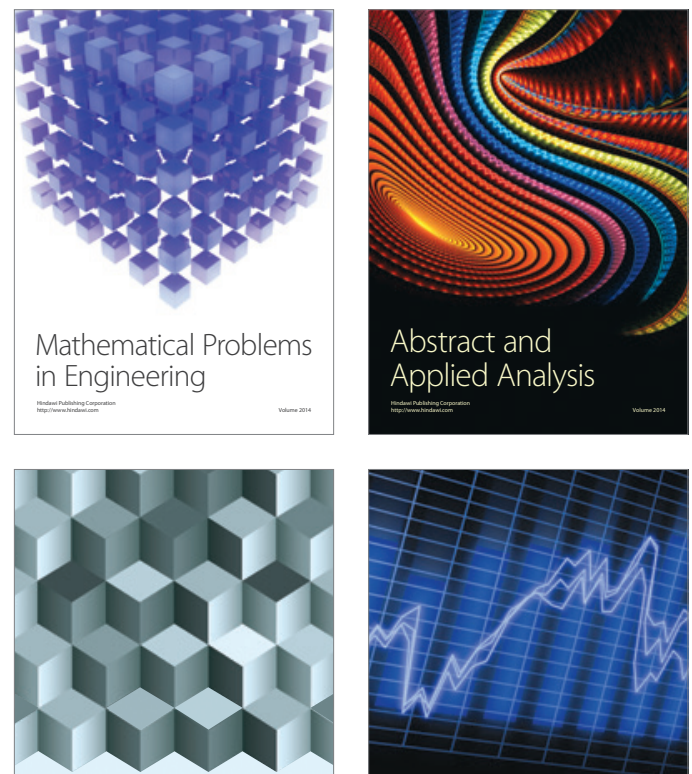

Journal of

Function Spaces

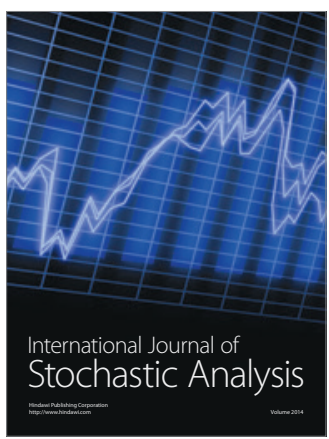

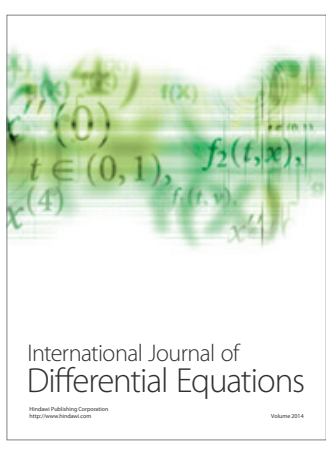
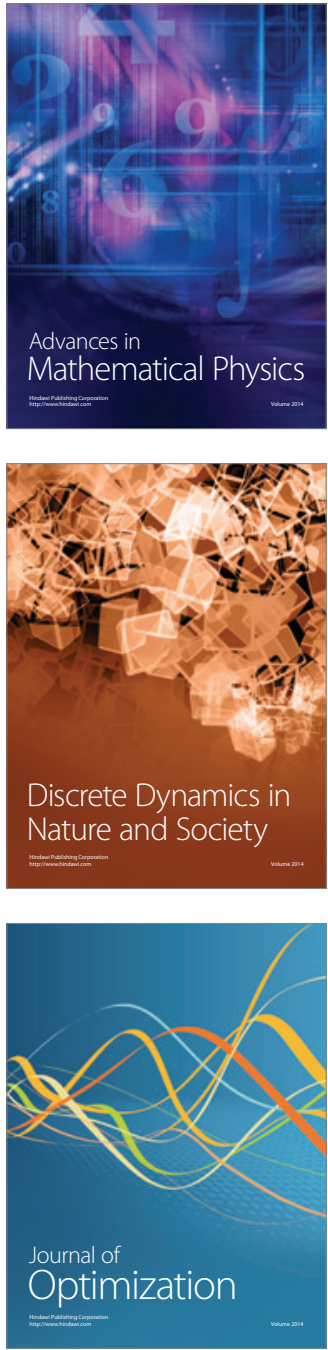